\title{
Microhydration of Biomolecules: Revealing the Native Structures by Cold Ion IR Spectroscopy.
}

\author{
Erik Saparbaev, Viktoriia Aladinskaia, Andrei Zviagin, Oleg V. Boyarkin*
}

Correspondence to: oleg.boiarkin@epfl.ch

\section{Table of Contents}

Experimental and chemicals

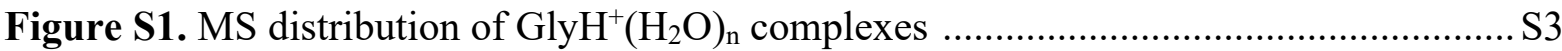

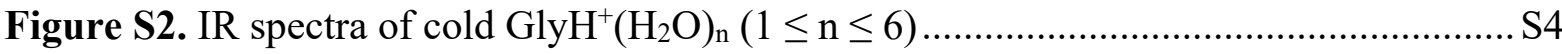

Table S1. Comparison of structures of the complexes produced by retaining and by condensation of water molecules for $\mathrm{GlyH}^{+}$and $\mathrm{Gly}_{3} \mathrm{H}^{+}$ 


\section{Experimental and chemicals}

The hydrated ions are produced in the gas phase from solution by a nano-electrospray ionization (n-ESI) source and transferred through a metal capillary and three consecutive inline molecular skimmers to a room temperature octupole ion trap for accumulation and thermalization. The thermalized ionic complexes are mass-selected by a quadrupole massfilter and guided into a cold octupole trap, ${ }^{24}$ which is kept at $6 \mathrm{~K}$. The stored in the trap complexes are cooled down to $\sim 10 \mathrm{~K}$ in collisions with He buffer gas ${ }^{24}$ and then irradiated by a pulse of IR light ( $6 \pm 2 \mathrm{~mJ} /$ pulse, $1 \mathrm{~cm}^{-1}$ spectral linewidth), produced by an optical parametric oscillator (OPO, Laser Vision). The absorption of the light results in the loss by a complex of a few water molecules. The remaining parent and the reduced fragment complexes are released from the trap and detected by a quadrupole mass spectrometer, which is tuned to transmit alternatively either one of the fragment or parent complexes. We average 10 measurements at each IR wavelength at a repetition rate of $10 \mathrm{~Hz}$. Each spectrum was recorded 2-3 times to ensure its reproducibility. A wavelength meter measures the wavenumber of the Nd:YAG laser and of the signal wave of the IR OPO, pumped by this laser, thus providing the wavenumber of the generated by the difference frequency mixing IR light with $\pm 0.2 \mathrm{~cm}^{-1}$ accuracy.

The spectra of $\mathrm{GlyH}^{+}\left(\mathrm{H}_{2} \mathrm{O}\right)_{\mathrm{n}}$ complexes have been measured by detecting the fragments $\mathrm{GlyH}^{+}\left(\mathrm{H}_{2} \mathrm{O}\right)_{\mathrm{m}}(\mathrm{m}=\mathrm{n}-2$ for $\mathrm{n} \geq 2$ and $\mathrm{m}=0$ for $\mathrm{n}=1)$; and the spectrum of $\mathrm{Gly}_{3} \mathrm{H}^{+}\left(\mathrm{H}_{2} \mathrm{O}\right)$ has been measured by detecting the bare peptide.

Glycine were purchased from ACROS Organics ( $>99 \%$ purity) and used without further purification. All the solvents of HPLC grade and acetic acid of $>99 \%$ purity are from Sigma-Aldrich. 


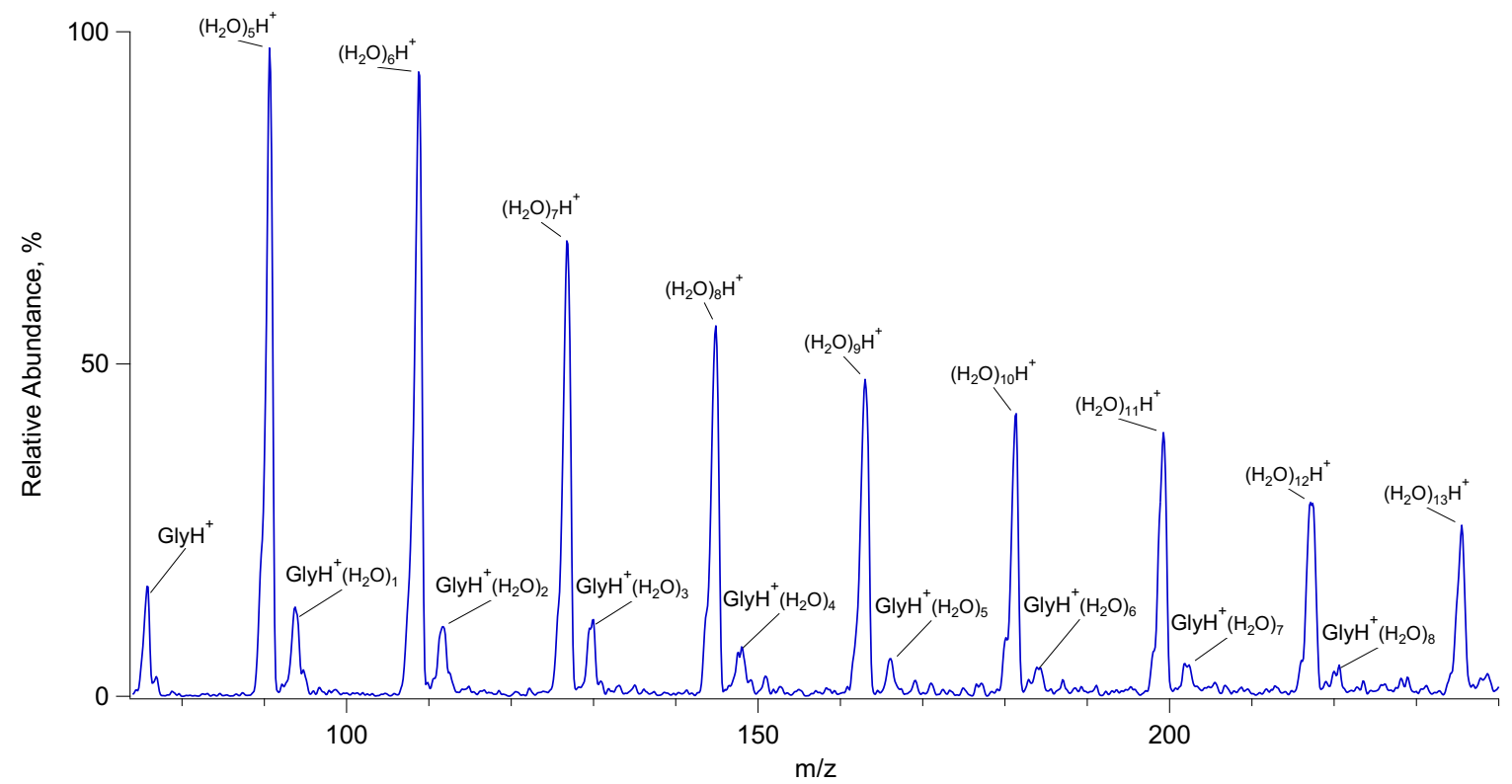

Figure S1. Mass-spectrum, showing distribution of $\mathrm{GlyH}^{+}\left(\mathrm{H}_{2} \mathrm{O}\right)_{\mathrm{n}}$ complexes, produced by a nano-electrospray under the conditions, not optimized for a particular number of water molecules $n$ in the complexes. Adjustments of voltages allow for some control of the complex size distribution. 


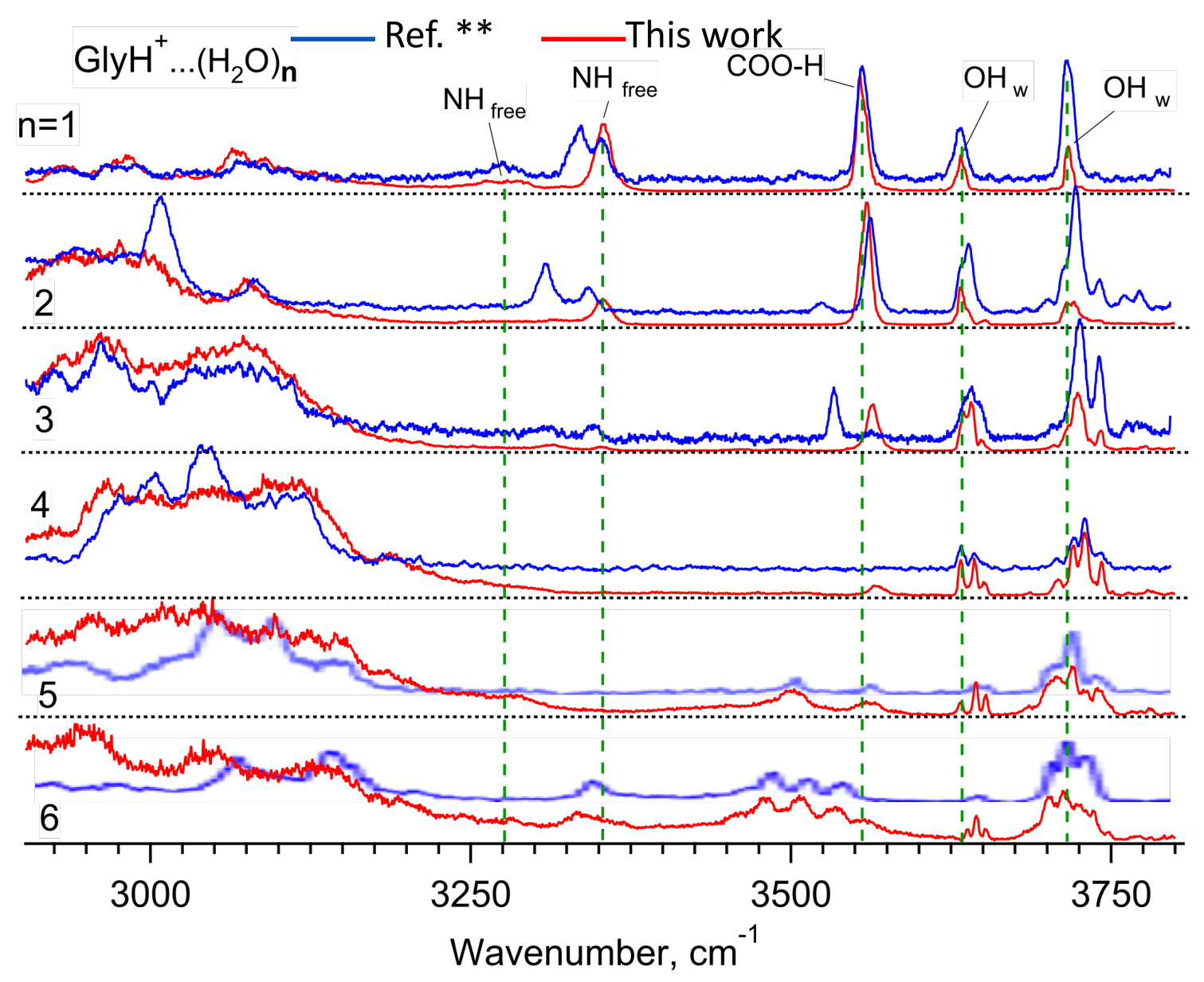

Figure S2. IR spectra of cold $\mathrm{GlyH}^{+}\left(\mathrm{H}_{2} \mathrm{O}\right)_{\mathrm{n}}(1 \leq \mathrm{n} \leq 6)$, produced directly from aqueous solution (red trace; this work) and by cryogenic condensation (blue trace, ref. ${ }^{21}$ ). Note, that the reproduced original blue traces were shifted by $-4 \mathrm{~cm}^{-1}$ to match to the manifold of the high-frequency transitions (free $\mathrm{OH}$-stretches of waters around $3700 \mathrm{~cm}^{-1}$ ) measured herein (red traces) with the accuracy of $\pm 0.2 \mathrm{~cm}^{-1}$. The blue traces are offset up for graphical clarity. 
Table S1. Comparison of structures for the complexes prepared by retaining from solution and by cryogenic condensation of water molecules. All the structures were calculated by the group of Garand (ref. ${ }^{16,17}$ ).

Condensed clusters Retained clusters

